\title{
Distinguishing numbers of Cartesian products of multiple complete graphs
}

\author{
Michael J. Fisher * \\ West Chester University, Department of Mathematics, West Chester, PA 19383, USA \\ Garth Isaak \\ Lehigh University, Department of Mathematics, Bethlehem, PA 18015, USA
}

Received 1 July 2010, accepted 3 October 2011, published online 1 February 2012

\begin{abstract}
We examine the distinguishing number of the Cartesian product of an arbitrary number of complete graphs. We show that for $u_{1} \leq \cdots \leq u_{d}$ the distinguishing number of the Cartesian product of complete graphs of these sizes is either $\left\lceil u_{d}^{1 / s}\right\rceil$ or $\left\lceil u_{d}^{1 / s}\right\rceil+1$ where $s=\Pi_{i=1}^{d-1} u_{i}$. In most cases, which of these values it is can be explicitly determined.
\end{abstract}

Keywords: Cartesian product, complete graph, distinguishing number.

Math. Subj. Class.: 05C78, 20B25, 68R10

\section{Introduction}

The distinguishing number of a graph is the minimum number of labels needed so that the only label preserving automorphism is the identity automorphism. The distinguishing number was introduced by Albertson and Collins in [2]. Albertson [1] observed that the distinguishing number of powers (with respect to Cartesian product) of complete graphs provides an upper bound on the distinguishing number of powers of prime graphs. (Formal definitions will be given in the next section.)

Building on results [3] that the hypercube has distinguishing number 2, Albertson [1] showed that that distinguishing number of the $d^{\text {th }}$ power of a complete graph is 2 when $d \geq 4$. This result was extended in [10] and [9] showing that Cartesian powers of complete graphs have distinguishing number 2 except for squares and cubes of $K_{2}$ and the square of $K_{3}$ all of which have distinguishing number 3 . We will look more generally at the distinguishing number of Cartesian products of complete graphs of different sizes.

\footnotetext{
* Corresponding author.

E-mail addresses: mfisher@wcupa.edu (Michael J. Fisher), gisaak@lehigh.edu (Garth Isaak)
} 
For products of graphs of different sizes, the distinguishing number of the Cartesian product of complete graphs on $u_{1}$ and $u_{2}$ vertices for $u_{1} \leq u_{2}$ was examined in [5] and [7]. It is either $\left\lceil u_{2}^{1 / u_{1}}\right\rceil$ or $\left\lceil u_{2}^{1 / u_{1}}\right\rceil+1$. In 'most' cases which value it is can be determined explicitly and in a few cases it can be determined using a recursion.

More generally one can talk about the distinguishing number of a group acting on a set as the minimum number of labels needed so that the only label preserving group element is the identity. When the group is the group of automorphisms of a graph and the set is the vertex set this is the distinguishing number of the graph. Chan [4] examined $S_{m} \times S_{n}$ acting on $[m] \times[n]$. When $m \neq n$ this is the distinguishing number of the Cartesian product of complete graphs of size $m$ and $n$ hence the results in the previous paragraph apply.

In this paper, we examine the distinguishing number of the Cartesian product of complete graphs of sizes $u_{1} \leq u_{2} \leq \cdots \leq u_{d}$. As a tool we will use the closely related distinguishing number of the group $S_{u_{1}} \times S_{u_{2}} \times \cdots \times S_{u_{d}}$ acting on $\left[u_{1}\right] \times\left[u_{2}\right] \times \cdots \times\left[u_{d}\right]$. These are identical problems when the $u_{i}$ are distinct. We will show the following. Except for $d=2$ with $u_{1}=u_{2}=2$ or $u_{1}=u_{2}=3$ and $d=3$ with $u_{1}=u_{2}=u_{3}=2$ these two distinguishing numbers are the same. This distinguishing number is either $\left\lceil u_{d}^{1 / s}\right\rceil$ or $\left\lceil u_{d}^{1 / s}\right\rceil+1$ where $s=\Pi_{i=1}^{d-1} u_{i}$. As with the case $d=2$, in 'most' cases which value it is can be determined explicitly and in a few cases it is can be determined using a recursion. Our proof uses induction via two basic reduction lemmas. The switching lemma extends a version for the Cartesian product of 2 complete graphs and reduces the sizes of the $u_{i}$. The collapsing lemma reduces the 'dimension', the number $d$ of factors.

A relatively simple argument for the lower bound, $\left\lceil u_{d}^{1 / s}\right\rceil$, is the following. For a $c$ coloring $f$ of $G \square K_{u}$ let $f_{v}$ be a coloring of $G$ given by $f_{v}=f(x, v)$. If $u>c^{s}$ there must be two vertices $v, v^{\prime}$ in $K_{n}$ with $f_{v}=f_{v^{\prime}}$. Then the automorphism that is constant on $G$ coordinates and transposing $v$ and $v^{\prime}$ is label preserving. Hence, the distinguishing number is at least $u^{1 / s}$ and, since it is integral, at least $\left\lceil u^{1 / s}\right\rceil$. Then apply this with $G=K_{u_{1}} \square \cdots \square K_{u_{d-1}}$. This approach is found in our Lemma 4.3 as well as earlier papers on distinguishing numbers of Cartesian products. The nice description above is suggested by an anonymous referee.

The upper bound $\left\lceil u^{1 / s}\right\rceil+1$ for Cartesian powers of general graphs follows from the upper bound observation noted above on powers of complete graphs (see also Observation 2.5 below and the comments surrounding it) and our upper bound in Corollary 5.1. When $s<u_{d}$ we do not need our Corollary 5.1 as it follows from the upper bound on Cartesian products of two complete graphs upper bound found independently in [5] and [7].

Hence, the main work in our proof covers the cases $u_{d} \leq s$. As $\left\lceil u_{d}^{1 / s}\right\rceil \leq 2$ for $0 \leq u_{d} \leq s$, we are establishing an upper bound of 3 on the distinguishing number in these cases. We work with general $c$ rather than $c=2$ needed for $u_{d} \leq s$ since, in addition, our proof establishes exactly which of the two bounds holds for 'most' cases of Cartesian products of complete graphs. It is also interesting to note that the determination of the exact value of the distinguishing number depends only on $u_{d}, u_{d-1}$ and the product $\Pi_{j=1}^{d-2} u_{j}$.

\section{Definitions and background}

We begin with basic definitions.

Definition 2.1 (Distinguishing Labeling). Given a set $V$ and a group $H$ acting on $V$, a labeling $\ell: X \rightarrow\{1,2, \ldots, c\}$ is $c$-distinguishing if only the identity preserves the labels. 
When the set $V$ is the vertex set of a graph and the group is the automorphisms of the graph we will call this a distinguishing labeling of the graph.

Definition 2.2 (Distinguishing Number). Given a set $V$ and a group $H$ acting on $V$, the distinguishing number $D_{H}(V)$ is the smallest $c$ such that there is a $c$-distinguishing labeling. For graph automorphisms, $\operatorname{Aut}(G)$ acting on a graph $G$ with vertex set $V$ we will write $D(G)$ instead of $D_{\operatorname{Aut}(G)}(V)$ and refer to the distinguishing number of the graph.

Definition 2.3. The Cartesian product of graphs $G_{1}$ and $G_{2}$ on vertex sets $V_{1}$ and $V_{2}$ respectively, denoted $G_{1} \square G_{2}$, is the graph with vertex set $V_{1} \times V_{2}$ and an edge between $\left(u_{1}, u_{2}\right)$ and $\left(v_{1}, v_{2}\right)$ if and only if either $u_{1}=v_{1}$ and $u_{2} v_{2}$ is an edge of $G_{2}$ or $u_{2}=v_{2}$ and $u_{1} v_{1}$ is an edge of $G_{1}$. Multiple powers are defined inductively $G_{1} \square G_{2} \square \cdots \square G_{d}=$ $\left(G_{1} \square \cdots \square G_{d-1}\right) \square G_{d}$.

The $d^{\text {th }}$ Cartesian power of a graph is the product of $d$ copies of the graph. That is, $G^{d}=G \square G^{d-1}$ with $G^{2}=G \square G$. A graph $G$ is prime with respect to Cartesian product if whenever $G=G_{1} \square G_{2}$, then either $G_{1}$ or $G_{2}$ is the trivial graph with a single vertex.

The operation $\square$ is associative and commutative. Graphs have unique prime factorizations $G=G_{1} \square \cdots \square G_{d}$ where each $G_{i}$ is prime. Graphs $G_{1}$ and $G_{2}$ are said to be relatively prime if they do not share a common factor.

In order to simplify notation, we will use the following terminology to distinguish the two closely related distinguishing labeling problems that we will examine.

For a Cartesian product $K_{u_{1}} \square K_{u_{2}} \square \cdots \square K_{u_{d}}$ of complete graphs, we can identify the vertices with $d$-tuples from $\left[u_{1}\right] \times\left[u_{2}\right] \times \cdots \times\left[u_{d}\right]$ with two vertices adjacent if and only if they agree on exactly $d-1$ coordinates. We can think of a $c$-labeling as an array of size $u_{1}, u_{2}, \ldots, u_{d}$ with entries from $[c]$. By the Imrich and Miller result noted below, automorphisms correspond to permutations of entries within each dimension along with 'transposes' of same size dimensions. For simplicity in notation, we will refer to this set and group of automorphisms as an array of size $u_{1}, u_{2}, \ldots, u_{d}$ and write $D\left(\operatorname{Array}\left(u_{1}, u_{2}, \ldots, u_{d}\right)\right)$ for the distinguishing number.

For distinguishing labelings of $S_{u_{1}} \times S_{u_{2}} \times \cdots \times S_{u_{d}}$ acting on $\left[u_{1}\right] \times\left[u_{2}\right] \times \cdots \times$ $\left[u_{d}\right]$, we can think of the elements as $d$-tuples from $\left[u_{1}\right] \times\left[u_{2}\right] \times \cdots \times\left[u_{d}\right]$. The group actions correspond to permutations of entries within each dimension. Unlike the previous paragraph, 'transposes' of same size dimensions do not occur. For simplicity in notation we will refer to this set and group of automorphisms as a grid of size $u_{1}, u_{2}, \ldots, u_{d}$ and write $D\left(\operatorname{Grid}\left(u_{1}, u_{2}, \ldots, u_{d}\right)\right)$ for the distinguishing number.

For completeness, we make the following trivial observations which follow from the fact that the group acting on the grids is a subgroup of that acting on the arrays.

Observation 2.4. If the array of size $u_{1}, u_{2}, \ldots, u_{d}$ has a distinguishing $c$-coloring, then the grid of size $u_{1}, u_{2}, \ldots, u_{d}$ has a distinguishing $c$-coloring. The distinguishing number of the grid of size $u_{1}, u_{2}, \ldots, u_{d}$ is at most the distinguishing number of the array of size $u_{1}, u_{2}, \ldots, u_{d}$. That is, $D\left(\operatorname{Grid}\left(u_{1}, u_{2}, \ldots, u_{d}\right)\right) \leq D\left(\operatorname{Array}\left(u_{1}, u_{2}, \ldots, u_{d}\right)\right)$.

As noted above, the distinguishing numbers of arrays and grids of the same size will be shown to be equal except in the following three cases: $d=2$ with $u_{1}=u_{2}=2$ or with $u_{1}=u_{2}=3$ and $d=3$ with $u_{1}=u_{2}=u_{3}=2$. In each of these small cases, it is straightforward to check that the grid distinguishing number is 2 and the array distinguishing number is 3. For arrays, the first two are noted in [3] and the third in [9]. 
For grids, the first two are noted in [4] and for the third, observe that the $[2] \times[2] \times[2]$ grid with with entries $a_{i, j, 1}$ given by $\left[\begin{array}{ll}1 & 2 \\ 2 & 2\end{array}\right]$ and entries $a_{i, j, 2}$ given by $\left[\begin{array}{ll}1 & 1 \\ 1 & 1\end{array}\right]$ is 2-distinguishing.

A result shown independently by Imrich and Miller (see [8]) is that if $\mathrm{G}$ is connected and $G=G_{1} \square \cdots \square G_{d}$ is its prime decomposition, then every automorphism of $\mathrm{G}$ is generated by the automorphisms of the factors and the transpositions of isomorphic factors. Using this we make the following observation which extends those of Albertson [1] and Imrich, Jerebic, and Klavžar [7]. As with the Observation 2.4, it follows as the group acting on the vertex set of the graph is a subgroup of that acting on the array.

Observation 2.5. If $G=G_{1} \square G_{2} \square \cdots \square G_{d}$ with the $G_{i}$ relatively prime and $\left|V\left(G_{i}\right)\right|=$ $u_{i}$, then $D\left(G_{1} \square G_{2} \square \cdots \square G_{d}\right) \leq D\left(\operatorname{Array}\left(u_{1}, u_{2}, \ldots, u_{d}\right)\right)$.

As a consequence of our results, we will see that $D\left(\operatorname{Array}\left(u_{1}, \ldots, u_{i-2}, u_{i-1}, u_{i}\right.\right.$, $\left.\left.u_{i+1}, \ldots, u_{s}\right)\right) \leq D\left(\operatorname{Array}\left(u_{1}, \ldots, u_{i-2},\left(u_{i-1} u_{i}\right), u_{i+1}, \ldots, u_{s}\right)\right)$. Thus, if the sizes of the factors in the prime factorization of $G_{1} \square G_{2} \square \ldots \square G_{d}$ are $u_{1}, u_{2}, \ldots, u_{d^{\prime}}$, then $D\left(G_{1} \square G_{2} \square \cdots \square G_{d}\right) \leq D\left(\operatorname{Array}\left(u_{1}, u_{2}, \ldots, u_{d^{\prime}}\right)\right)$. Hence, we can drop the relatively prime assumption in Observation 2.5.

We will also use the following definitions and notation. Examples of switching and collapsing in 2 dimensions are described in the next section.

For automorphisms of grids we will write $\left(\pi_{1}, \pi_{2}, \ldots, \pi_{d}\right)$ where $\pi_{i}$ is a permutation of $\left[u_{i}\right]$. For arrays we will write automorphisms as $\left(\sigma ; \pi_{1}, \ldots, \pi_{d}\right)$ where the $\pi_{i}$ are as for grids and $\sigma$ is a permutation of $[d]$ (such that only positions with equal sizes can be permuted). We will apply the $\pi_{i}$ first and then permute the dimensions.

Definition 2.6 (Switching). For a $c$-labeling $A$ with entries $a\left(i_{1}, i_{2}, \ldots, i_{d}\right)$ of an array (grid) of size $u_{1}, u_{2}, \ldots, u_{d}$, the dimension $i$ subarrays are the $(d-1)$ dimensional arrays $A_{k}$ of size $\left(u_{1}, \ldots, u_{i-1}, u_{i+1}, \ldots, u_{d}\right)$ given by $a_{k}\left(j_{1}, \ldots, j_{i-1}, j_{i+1}, \ldots, j_{d}\right)=$ $a\left(j_{1}, \ldots, j_{i-1}, k, j_{i+1}, \ldots, j_{d}\right)$ for $k=1,2, \ldots, u_{i}$.

If $A$ is a $c$-labeling of an array of size $u_{1}, u_{2}, \ldots, u_{d}$ and the dimension $i$ subarrays are distinct, then the dimension $i$ complement $A_{i}^{*}$ is the size $u_{1}, \ldots, u_{i-1}, c^{p}-u_{i}, u_{i+1}, \ldots, u_{d}$ array where $p=u_{1} \cdots u_{i-1} u_{i+1} \cdots u_{d}$, whose set of dimension $i$ subarrays is the set of all $c$ labelings of arrays of size $\left(u_{1}, \ldots, u_{i-1}, u_{i+1}, \ldots, u_{t}\right)$ that do not appear as dimension $i$ subarrays of $A$.

For example, in two dimensions the dimension 1 subarrays are the rows and the dimension 2 subarrays are the columns. Also, in two dimensions, the dimension 2 complement $A_{2}^{*}$ is the size $\left(u_{1}, c^{u_{1}}-u_{2}\right)$ array consisting of all 'possible' columns (size $u_{1}$ strings with entries from $[c]$ ) that do not appear in $A$.

Technically, the complement would depend on the ordering of the complementary subarrays but as this will not affect what we do we will assume some fixed ordering and refer to 'the' complement.

Definition 2.7 (Collapsing). For a $c$-labeling $A$ with entries $a\left(i_{1}, i_{2}, \ldots, i_{d}\right)$ of an array of size $u_{1}, u_{2}, \ldots, u_{d}$ and $S=\{k+1, \ldots, d\}$ the array $A^{S}$ obtained by collapsing the dimensions in $S$ has $d-|S|+1$ dimensions with sizes $\left(u_{1}, u_{2}, \ldots, u_{k}, q\right)$ where $q=$ $u_{k+1} \cdots u_{d}$. It is defined as follows: Let $g$ be any bijection from $\left[u_{k+1}\right] \times \cdots \times\left[u_{d}\right]$ to $\left[u_{k+1} \cdots u_{d}\right]$. The entries of $A^{S}$ are then $a^{S}\left(j_{1} \ldots, j_{k}, g\right)=a\left(j_{1}, \ldots, j_{k}, j_{k+1}, \ldots, j_{d}\right)$ where $g=g\left(j_{k+1}, \ldots, j_{d}\right)$. 
For any subset $S \subset[d]$ of indices, the array $A_{S}$ obtained by collapsing the dimensions in $S$ is defined in a similar manner and has $d-|S|+1$ dimensions with sizes $u_{i}$ for $i \notin S$ and one dimension of size $\Pi_{i \in S} u_{i}$.

Technically, the collapse would depend on the choice of $g$ but as this will not affect what we do we will assume some fixed $g$ and refer to 'the' collapse.

\section{Reduction lemmas}

We will make use of two basic lemmas to relate distinguishing labelings of one size to another. This will allow for induction to determine values of $c$ for which arrays and grids of given sizes have distinguishing $c$-labelings.

Consider a $c$-labeling $A$ of the size $u_{1}, u_{2}$ grid with distinct columns. The dimension 2 complement $A_{2}^{*}$ has size $u_{1}, c^{u_{1}}-u_{2}$ and consists of all $c^{u_{1}}-u_{2}$ possible columns that do not appear in $A$. An automorphism $\left(\pi_{1}, \pi_{2}\right)$ of $A$ that preserves labels maps the columns of $A$ to columns of $A$ under $\pi_{1}$. Hence, applying $\pi_{1}$ to $A_{2}^{*}$ must map columns of $A_{2}^{*}$ to columns of $A_{2}^{*}$. So we can find a permutation $\pi_{2}^{\prime}$ of $\left[c^{u_{1}}-u_{2}\right]$ so that $\left(\pi_{1}, \pi_{2}^{\prime}\right)$ is a automorphism of $A_{2}^{*}$ that preserves labels. So, we observe that there is a distinguishing labeling of the size $u_{1}, u_{2}$ grid if and only if there is a distinguishing labeling of the size $u_{1}, c^{u_{1}}-u_{2}$ grid. For arrays, we get the same result except that when $u_{1}=u_{2}$ there is the possibility of an automorphism that transposes rows and columns of $A$ that does not correspond to an automorphism of $A_{2}^{*}$. So, in this case, we get only that if there is a distinguishing labeling of the size $u_{1}, c^{u_{1}}-u_{2}$ array and $u_{1} \neq u_{2}$, then there is a distinguishing labeling of the size $u_{1}, u_{2}$ array. This idea will be presented as the switching lemma below.

The idea in the switching lemma (in two dimensions) below has appeared in a number of earlier papers on distinguishing number. It appears in some form (at least) in [4], [5], [7] [9] and [6]. We use the name switching lemma given in [7].

For simplicity in notation, we state the following lemma for switching in dimension $d$. By permuting the labels on the dimensions, a similar statement holds for switching on any dimension. We state versions for both grids and arrays. The proofs are essentially the same as in the two dimensional case. For completeness, we give a proof for the grid version.

Lemma 3.1 (Grid Switching Lemma). Let $u_{1} \leq u_{2} \leq \cdots \leq u_{d}$ with $u_{d}<c^{p}$ where $p=u_{1} u_{2} \cdots u_{d-1}$. There is a $c$-distinguishing labeling of a grid of size $u_{1}, u_{2}, \ldots, u_{d}$ if and only if there is a $c$-distinguishing labeling of a grid of size $u_{1}, u_{2}, \ldots, u_{d-1}, c^{p}-u_{d}$.

Proof. Consider a $c$-labeling $A$ of the size $u_{1}, u_{2}, \ldots, u_{d}$ grid with distinct $(d-1)$-dimensional subgrids. The dimension $d$ complement $A_{d}^{*}$ has size $u_{1}, u_{2}, \ldots, c^{p}-u_{d}$ and consists of all $c^{p}-u_{d}$ possible $(d-1)$-dimensional subgrids that do not appear in $A$. An automorphism $\left(\pi_{1}, \pi_{2}, \ldots, \pi_{m}\right)$ of $A$ that preserves labels maps the $(d-1)$-dimensional subgrids of $A$ to the $(d-1)$-dimensional subgrids of $A$ under $\pi_{s u b}=\left(\pi_{1}, \pi_{2}, \ldots, \pi_{d-1}\right)$. Hence, applying $\pi_{s u b}$ to $A_{d}^{*}$ must map the $(d-1)$-dimensional subgrids of $A_{d}^{*}$ to the $(d-1)$-dimensional subgrids of $A_{d}^{*}$. So, we can find a permutation $\pi_{d}^{\prime}$ of $\left[c^{p}-u_{d}\right]$ so that $\left(\pi_{1}, \pi_{2}, \ldots, \pi_{d-1}, \pi_{d}^{\prime}\right)$ is an automorphism of $A_{d}^{*}$ that preserves labels.

We state the following without proof as it is identical to the grid switching lemma.

Lemma 3.2 (Array Switching Lemma). If there is a $c$-distinguishing labeling of an array of size $u_{1}, u_{2}, \ldots, u_{m-1}, c^{p}-u_{m}$ where $p=u_{1} u_{2} \cdots u_{m-1}$ and $u_{m} \neq u_{k}$ for all $k=$ $1,2, \ldots, m-1$, then there is a $c$-distinguishing labeling of an array of size $u_{1}, u_{2}, \ldots, u_{m}$. 
Our second reduction lemma, which we will call the collapsing lemma, allows a reduction in the number of dimensions. It is based on the following simple observation. Consider a $c$-labeling $A$ of the size $u_{1}, u_{2}$ array. We can write the rows one after another in a string of length $u_{1} u_{2}$ (i.e., 1-dimensional array $A_{\{1,2\}}$ of size $\left.u_{1} u_{2}\right)$. An automorphism $\left(\pi_{1}, \pi_{2}\right)$ of $A$ permutes entries of $A$ and hence there is a corresponding permutation $\pi_{1,2}$ of the entries of $A_{\{1,2\}}$. Thus, if $A_{\{1,2\}}$ is a distinguishing $c$-labeling then so is $A$. Note that the converse does not hold (indeed in two dimensions the entries need to be distinct for $A_{\{1,2\}}$ to be distinguishing).

For higher dimensions, we need to be a little careful with equal sizes. For example, consider a $c$-labeling $A$ of size $\left(u_{1}, u_{2}, u_{3}\right)$ with $u_{1}=u_{2}$. An automorphism of $A$ that transposes dimensions 1 and 2 does not necessarily correspond to an automorphism of $A_{\{2,3\}}$, obtained by collapsing dimensions 2 and 3 . So, if $u_{i}=u_{j}$, then we must either include both in the dimension we collapse or exclude both.

As with the switching lemma, for simplicity in notation we state the collapsing lemma for collapsing dimensions $k+1, \ldots, d$. By permuting labels of the dimensions, a similar statement holds for collapsing any set $S$ as long as $u_{i}=u_{j}$ implies either $i, j \in S$ or $i, j \notin S$.

Lemma 3.3 (Collapsing Lemma). If there is a distinguishing $c$-labeling of the $(k+1)$ dimensional array of size $\left(u_{1}, u_{2}, \ldots, u_{k},\left(u_{k+1} \cdots u_{d}\right)\right)$ and for $i \leq k$ and $j>k$ we have $u_{i} \neq u_{j}$, then there is a distinguishing $c$-labeling of the $d$ dimensional array of size $\left(u_{1}, u_{2}, \ldots, u_{k}, u_{k+1}, \ldots, u_{d}\right)$.

Proof. We prove the contrapositive. Let $A$ be a $c$-labeling of the $d$-dimensional array of size $\left(u_{1}, u_{2}, \ldots, u_{k}, u_{k+1}, \ldots, u_{d}\right)$ and define $A^{S}$ as in definition 2.7 with $S=\{k+1, \ldots, d\}$.

Let $A^{S}$ be a distinguishing $c$-labeling of $\left(u_{1}, u_{2}, \ldots, u_{k},\left(u_{k+1} \cdots u_{d}\right)\right)$ and let $A$ be the associated $c$-labeling of $\left(u_{1}, u_{2}, \ldots, u_{k}, u_{k+1}, \ldots, u_{d}\right)$. An automorphism $\left(\sigma ; \pi_{1}, \pi_{2}, \ldots\right.$, $\left.\pi_{d}\right)$ of $A$ can be used to define an automorphism $\left(\sigma^{\prime} ; \pi_{1}, \ldots, \pi_{k}, \pi_{k+1}\right)$ of $A^{S}$ in the 'obvious' way. We have $\sigma^{\prime}$ the same as $\sigma$ on $\{1,2, \ldots, k\}$ with $k+1$ fixed. The automorphism produces some permutation of the entries in dimensions $k+1, \ldots, d$ which we call $\pi_{k+1}^{\prime}$. It is straightforward to see that we get an automorphism of $A^{S}$. Hence, if $A$ is not a distinguishing $c$-labeling of $\left(u_{1}, u_{2}, \ldots, u_{k}, u_{k+1}, \ldots, u_{t}\right)$, then $A^{S}$ is not a distinguishing $c$-labeling of $\left(u_{1}, u_{2}, \ldots, u_{k},\left(u_{k+1} \cdots u_{t}\right)\right)$.

We observe that there is a corresponding collapsing lemma for grids without the size conditions. We do not need this in what follows so we will not formally state it.

\section{Distinguishing labelings}

In this section we state a theorem describing those values of $c$ for which there are distinguishing labelings of arrays and grids of a given size. We first give lemmas providing upper and lower bounds.

The following lemma will be used several times in the proof of Lemma 4.2.

Lemma 4.1. Let $d$ be an integer greater than 3. If the array $\left(u_{1}, u_{2}, \ldots, u_{d-1}\right)$ has a distinguishing 2-labeling and if $u_{d} \leq u_{1} u_{2} \cdots u_{d-1}+1$, then $\left(u_{1}, u_{2}, \ldots, u_{d-1}, u_{d}\right)$ also has a distinguishing 2-labeling. 
Proof. Suppose that $\left(u_{1}, u_{2}, \ldots, u_{d-1}\right)$ has a distinguishing 2-labeling; call it $\ell$. We define the weight of a generic 2-labeling to be the total number of times the label one is used in the labeling. Because there are $u_{1} u_{2} \cdots u_{d-1}+1$ 2-labelings of $\left(u_{1}, u_{2}, \ldots, u_{d-1}\right)$ with different weights, we can construct a distinguishing 2-labeling of $\left(u_{1}, u_{2}, \ldots, u_{d-1}, u_{d}\right)$ by taking $\ell$ together with any $u_{d}-1$ of the other remaining $u_{1} u_{2} \cdots u_{d-1}$ 2-labelings of $\left(u_{1}, u_{2}, \ldots, u_{d-1}\right)$ with different weights.

Lemma 4.2. For integers $c \geq 2, d \geq 3$ and $1 \leq u_{1} \leq u_{2} \leq \cdots \leq u_{d}$ with $u_{d} \leq$ $c^{u_{1} u_{2} \cdots u_{d-1}}-\left\lceil\frac{\log _{c} u_{d-1}}{u_{1} u_{2} \cdots u_{d-2}}\right\rceil-1$ there is a distinguishing $c$-labeling of the $\left(u_{1}, u_{2}, \ldots, u_{d}\right)$ array.

Proof. The proof will proceed by induction on the number $d$ of dimensions and by induction on $u_{d}$. We will use the case $d=2$ as the basis. For $d=2$ and $u_{1} \leq u_{2}$, if $u_{2} \leq c^{u_{1}}-\left\lceil\log _{c} u_{1}\right\rceil-1$, then the $\left(u_{1}, u_{2}\right)$ array has a distinguishing $c$-labeling ([5], [7]). This corresponds to the formula above if we interpret the product $u_{1} \cdots u_{d-2}$ as an empty product equal to 1 when $d=2$.

We will establish the result for $d=3$ and $d=4$ separately and then proceed to the case $d \geq 5$. For each $d$, several cases are needed to determine where collapsing can occur.

$d=3$ : We first establish base cases for induction on $u_{3}$. Note that the collapsing lemma implies that $(2,2,3)$ has a distinguishing 2-labeling (collapsing the first two terms we have a size $(4,3)$ array which has a distinguishing 2-labeling). It is easy to verify that the two 2-labelings of $(3,3)$

$$
\left[\begin{array}{lll}
2 & 2 & 2 \\
1 & 2 & 1 \\
1 & 2 & 2
\end{array}\right] \text { and }\left[\begin{array}{ccc}
1 & 1 & 2 \\
2 & 1 & 1 \\
2 & 2 & 1
\end{array}\right]
$$

provide us with an explicit distinguishing 2-labeling of $(2,3,3)$. The first array giving the $a_{1, j, k}$ entries and the second the $a_{2, j, k}$ entries. Finally, a result of Klavžar and Zhu [10] tells us that $(3,3,3)$ has a distinguishing 2-labeling.

Case 1: $\left(u_{1}<u_{2}<u_{3}\right)$

(i) If $u_{1} u_{2}<u_{3}$, then $\left(\left(u_{1} u_{2}\right), u_{3}\right)$ has a distinguishing $c$-labeling if $u_{3} \leq c^{u_{1} u_{2}}-$ $\left\lceil\log _{c} u_{1} u_{2}\right\rceil-1$. By the collapsing lemma, $\left(u_{1}, u_{2}, u_{3}\right)$ also has a distinguishing $c$-labeling.

If $c^{u_{1} u_{2}}-\left\lceil\log _{c} u_{1} u_{2}\right\rceil-1<u_{3} \leq c^{u_{1} u_{2}}-\left\lceil\frac{\log _{c} u_{2}}{u_{1}}\right\rceil-1$, then switch in dimension 3 and consider $\left(u_{1}, u_{2}, z\right)$ where $\left\lceil\frac{\log _{c} u_{2}}{u_{1}}\right\rceil+1 \leq z \leq\left\lceil\log _{c} u_{1} u_{2}\right\rceil<u_{3}$.

(a) If $2 \leq u_{1}<u_{2} \leq z$, then, as $z<u_{3} \leq c^{u_{1} u_{2}}-\left\lceil\frac{\log _{c} u_{2}}{u_{1}}\right\rceil-1,\left(u_{1}, u_{2}, z\right)$ has a distinguishing $c$-labeling by induction on $u_{3}$. Thus, by the switching lemma, $\left(u_{1}, u_{2}, u_{3}\right)$ also has a distinguishing $c$-labeling.

(b) If $u_{1}<z<u_{2}$, then it is always true that $u_{2} \leq c^{z u_{1}}-\left\lceil\frac{\log _{c} z}{u_{1}}\right\rceil-1$. [ $z \geq$ $\frac{\log _{c} u_{2}}{u_{1}}+1$ implies that $c^{z u_{1}} \geq u_{2} c^{u_{1}}$. Thus, $c^{z u_{1}} \geq u_{2} c^{u_{1}}$ and $z<u_{2}$ imply that $u_{2} \leq u_{2} c^{u_{1}}-u_{2}-1 \leq c^{z u_{1}}-\left\lceil\frac{\log _{c} z}{u_{1}}\right\rceil-1$.] Hence, by induction on $u_{3}$ and the switching lemma, $\left(u_{1}, u_{2}, u_{3}\right)$ has a distinguishing $c$-labeling. 
(c) If $z<u_{1}<u_{2}$, then it is always true that $u_{2} \leq c^{z u_{1}}-\left\lceil\frac{\log _{c} u_{1}}{z}\right\rceil-1$. [z $\geq$ $\frac{\log _{c} u_{2}}{u_{1}}+1$ implies that $c^{z u_{1}} \geq u_{2} c^{u_{1}}$. Thus, $c^{z u_{1}} \geq u_{2} c^{u_{1}}$ implies that $u_{2} \leq$ $u_{2} c^{u_{1}}-u_{2}-1 \leq u_{2} c^{u_{1}}-u_{1}-1 \leq c^{z u_{1}}-\left\lceil\frac{\log _{c} u_{1}}{z}\right\rceil-1$.] Hence, by induction on $u_{3}$ and the switching lemma, $\left(u_{1}, u_{2}, u_{3}\right)$ has a distinguishing $c$-labeling.

(d) If $u_{1}=z<u_{2}$ and $u_{1}^{2}<u_{2}$, then it is always true that $u_{2} \leq c^{z u_{1}}-\left\lceil\frac{\log _{c} z}{z}\right\rceil-1=$ $c^{z u_{1}}-2$. $\left[z \geq \frac{\log _{c} u_{2}}{u_{1}}+1\right.$ implies that $c^{z u_{1}} \geq u_{2} c^{u_{1}}$. Thus, $c^{z u_{1}} \geq u_{2} c^{u_{1}}$ implies that $u_{2} \leq u_{2} c^{u_{1}}-2 \leq c^{z u_{1}}-2$.] It follows that $\left(u_{1}^{2}, u_{2}\right)$ has a distinguishing $c$-labeling. Thus, by the collapsing lemma, so does $\left(u_{1}, z, u_{2}\right)$.

(e) If $u_{1}=z<u_{2}$ and $u_{1}^{2}=u_{2}$, then $\left(u_{2}, u_{2}\right)$ has a distinguishing 2-labeling via the $d=2$ result. Thus, by the collapsing lemma, $\left(u_{1}, z, u_{2}\right)$ also has a distinguishing 2-labeling. Hence, $\left(u_{1}, z, u_{2}\right)$ also has a distinguishing $c$-labeling.

(f) Finally, if $u_{1}=z<u_{2}$ and $u_{2}<u_{1}^{2}$, then it is always true that $u_{1}^{2} \leq 2^{u_{2}}-$ $\left\lceil\log _{2} u_{2}\right\rceil-1$. [It is easy to check that this inequality holds for $u_{2}=3,4$. For $u_{2} \geq 5$, a simple inductive argument implies that $u_{2}^{2} \leq 2^{u_{2}}-u_{2}-1$. The desired inequality now follows.] It follows that $\left(u_{2}, u_{1}^{2}\right)$ has a distinguishing 2-labeling. Thus, by the collapsing lemma, so does $\left(u_{1}, z, u_{2}\right)$. Therefore, $\left(u_{1}, z, u_{2}\right)$ also has a distinguishing $c$-labeling.

Thus, in any case, $\left(u_{1}, u_{2}, u_{3}\right)$ also has a distinguishing $c$-labeling by the switching lemma.

(ii) If $u_{3} \leq u_{1} u_{2}$, then since $u_{1} u_{2} \leq 2^{u_{1} u_{2}}-\left\lceil\frac{\log _{2} u_{2}}{u_{1}}\right\rceil-1$ always holds, $\left(u_{1}, u_{2}, u_{3}\right)$ has a distinguishing 2-labeling by induction on dimension and by the collapsing lemma. [ $u_{1}<u_{2}$ implies that $u_{2}=u_{1}+m$ for some $m \geq 1$. Using first semester calculus, one can show that the function $f(x)=2^{x^{2}+m x}-1-x^{2}-(m+1) x-m$ is strictly increasing for $x \geq 2$ (and $m$ fixed). Since $f(2)>0$, the desired inequality now follows.]

Case 2: $\left(u_{1}<u_{2}=u_{3}\right)$

First note that it has already been shown that $(2,3,3)$ has a distinguishing 2-labeling. Next, recall that it is known (by the $d=2$ case) that if $4 \leq u_{2}=u_{3}$, then $\left(u_{2}, u_{3}\right)$ has a distinguishing 2-labeling. Thus, Lemma 4.1 implies that $\left(u_{1}<u_{2}=u_{3}\right)$ also has a distinguishing 2-labeling.

Case 3: $\left(u_{1}=u_{2}=u_{3}\right)$

This case follows from results in [9].

Case 4: $\left(u_{1}=u_{2}<u_{3}\right)$, where $u_{2}+1 \leq u_{3} \leq c^{u_{2}^{2}}-\left\lceil\frac{\log _{c} u_{2}}{u_{1}}\right\rceil-1$

(i) For $\left(u_{1}=u_{2}<u_{2}+\varepsilon\right), 1 \leq \varepsilon \leq u_{2}^{2}-u_{2}$, we collapse and consider $\left(u_{2}+\varepsilon, u_{2}^{2}\right)$. As we will show, $u_{2}^{2} \leq 2^{u_{2}+\varepsilon}-\left\lceil\log _{2}\left(u_{2}+\varepsilon\right)\right\rceil-1$ holds for all $u_{2} \geq 2$. Thus, $\left(u_{2}+\varepsilon, u_{2}^{2}\right)$ has a distinguishing 2-labeling by induction on dimension and hence $\left(u_{1}=u_{2}<u_{2}+\varepsilon\right)$ has a distinguishing 2-labeling by the collapsing lemma.

$\left[u_{2}^{2} \leq 2^{u_{2}+\varepsilon}-\left\lceil\log _{2}\left(u_{2}+\varepsilon\right)\right\rceil-1\right.$ can be verified by hand for the cases $u_{2}=2,3,4$. For $u_{2} \geq 5$, one can prove by induction that $u_{2}^{2} \leq 2^{u_{2}+1}-u_{2}^{2}-1$. Our result then follows.] 
(ii) For $\left(u_{1}=u_{2}<u_{2}^{2}+1\right)$ through $\left(u_{1}=u_{2}<c^{u_{2}^{2}}-\left\lceil\log _{c} u_{2}^{2}\right\rceil-1\right)$, we collapse and look at $\left(u_{2}^{2}, z\right), u_{2}^{2}+1 \leq z \leq c^{u_{2}^{2}}-\left\lceil\log _{c} u_{2}^{2}\right\rceil-1$. By induction on dimension, $\left(u_{2}^{2}, z\right)$ has a distinguishing $c$-labeling.

(iii) Finally, we see by the switching lemma that $\left(u_{1}=u_{2}<c^{u_{2}^{2}}-\left\lceil\log _{c} u_{2}^{2}\right\rceil\right)$ through $\left(u_{1}=u_{2}<c^{u_{2}^{2}}-\left\lceil\frac{\log _{c} u_{2}}{u_{2}}\right\rceil-1\right)$ all have a distinguishing $c$-labeling. Note that one case not covered here is $(2,2,14)((2,2,2)$ does not have a distinguishing 2-labeling). However, since $(4,14)$ has a distinguishing 2-labeling, the collapsing lemma shows that $(2,2,14)$ does too.

$d=4$ : The case $(2,2,2,2)$ will serve as the basis step for induction on $u_{4}$.

Case 1: $\left(u_{1}<u_{2}<u_{3}<u_{4}\right)$

(i) If $u_{1} u_{2} \leq u_{3}$, then, by induction on dimension, $\left(u_{1} u_{2}, u_{3}, u_{4}\right)$ has a distinguishing $c$-labeling if $u_{4} \leq c^{u_{1} u_{2} u_{3}}-\left\lceil\frac{\log _{c} u_{3}}{u_{1} u_{2}}\right\rceil-1$.

(ii) If $u_{3}<u_{1} u_{2}=u_{4}$, then we use Lemma 4.1 to build a distinguishing 2-labeling for $\left(u_{3}, u_{1} u_{2}, u_{4}\right)$ from a distinguishing 2-labeling of $\left(u_{1} u_{2}, u_{4}\right)$.

(iii) If $u_{3}<u_{1} u_{2}<u_{4}$, then $\left(u_{3}, u_{1} u_{2}, u_{4}\right)$ has a distinguishing $c$-labeling if $u_{4} \leq$ $c^{u_{1} u_{2} u_{3}}-\left\lceil\frac{\log _{c} u_{1} u_{2}}{u_{3}}\right\rceil-1$. If $c^{u_{1} u_{2} u_{3}}-\left\lceil\frac{\log _{c} u_{1} u_{2}}{u_{3}}\right\rceil-1<u_{4} \leq c^{u_{1} u_{2} u_{3}}-\left\lceil\frac{\log _{c} u_{3}}{u_{1} u_{2}}\right\rceil-1$, then we switch in the last dimension and look at $\left\lceil\frac{\log _{c} u_{3}}{u_{1} u_{2}}\right\rceil+1 \leq z<\left\lceil\frac{\log _{c} u_{1} u_{2}}{u_{3}}\right\rceil+1$. Note that the last string of inequalities is equivalent to $2 \leq z<3$. Thus, the only value of $z$ that we need to check is $z=2$.

To decide whether or not $\left(2, u_{3}, u_{1} u_{2}\right)$ has a distinguishing $c$-labeling, we switch in the second dimension and look at $\left(2, c^{2 u_{1} u_{2}}-u_{3}, u_{1} u_{2}\right)$. Since $u_{3}<u_{1} u_{2}$, we have $u_{1} u_{2} \leq c^{2 u_{1} u_{2}}-u_{3}$. Now, since $u_{3} \geq\left\lceil\log _{c} u_{2}\right\rceil+1 \geq\left\lceil\frac{\log _{c} u_{1} u_{2}}{2}\right\rceil+1$, it follows that $c^{2 u_{1} u_{2}}-u_{3} \leq c^{2 u_{1} u_{2}}-\left\lceil\frac{\log _{c} u_{1} u_{2}}{2}\right\rceil-1$. Hence, $\left(2, u_{1} u_{2}, c^{2 u_{1} u_{2}}-u_{3}\right)$ has a distinguishing $c$-labeling. Therefore, $\left(2, u_{3}, u_{1} u_{2}\right)$ does too.

(iv) If $u_{4}<u_{1} u_{2}$, then it is easily seen that $u_{1} u_{2} \leq 2^{u_{3} u_{4}}-\left\lceil\frac{\log _{2} u_{4}}{u_{3}}\right\rceil-1$. $\left[u_{1} u_{2}<\right.$ $u_{3} u_{4}$ and $u_{4}<u_{1} u_{2}$ imply the desired inequality.] Hence, $\left(u_{3}, u_{4}, u_{1} u_{2}\right)$ has a distinguishing 2-labeling.

Case 2: $\left(u_{1} \leq u_{2}<u_{3}=u_{4}\right)$

(i) If $u_{1} u_{2}<u_{3}$, then $u_{4} \leq 2^{u_{1} u_{2} u_{3}}-\left\lceil\frac{\log _{2} u_{3}}{u_{1} u_{2}}\right\rceil-1$ always holds. [One can show by induction on $u_{4}$ that $2 u_{4} \leq 2^{u_{1} u_{2} u_{4}}-1$. The desired inequality follows from the last statement.] Hence, by the collapsing lemma, $\left(u_{1}, u_{2}, u_{3}, u_{4}\right)$ has a distinguishing 2-labeling.

(ii) Similarly, if $u_{3} \leq u_{1} u_{2}$, then $u_{4}=u_{3} \leq u_{1} u_{2}<u_{4}^{2} \leq 2^{u_{4}^{2}}-2$ always holds. Hence, by the collapsing lemma, $\left(u_{1}, u_{2}, u_{3}, u_{4}\right)$ has a distinguishing 2-labeling.

Case 3: $\left(u_{1}<u_{2}=u_{3}<u_{4}\right)$ 
(i) If $u_{2}^{2}<u_{4}$, then $\left(u_{1}, u_{2}, u_{3}, u_{4}\right)$ has a distinguishing $c$-labeling, by the collapsing lemma, if $u_{4} \leq c^{u_{1} u_{2}^{2}}-\left\lceil\frac{\log _{c} u_{2}^{2}}{u_{1}}\right\rceil-1$. If $c^{u_{1} u_{2}^{2}}-\left\lceil\frac{\log _{c} u_{2}^{2}}{u_{1}}\right\rceil-1<u_{4} \leq c^{u_{1} u_{2}^{2}}-$ $\left\lceil\frac{\log _{c} u_{3}}{u_{1} u_{2}}\right\rceil-1$, then we switch in the last dimension and look at $\left\lceil\frac{\log _{c} u_{3}}{u_{1} u_{2}}\right\rceil+1 \leq z<$ $\left\lceil\frac{\log _{c} u_{2}^{2}}{u_{1}}\right\rceil+1$. The last string of inequalities implies that $2 \leq z<2 u_{2}$.

(a) If $2 \leq z \leq u_{2}$, then $\left(u_{1}, z, u_{2}, u_{3}\right)$ (or $\left(z, u_{1}, u_{2}, u_{3}\right)$ ) has a distinguishing 2labeling as long as $u_{3} \leq 2^{z u_{1} u_{2}}-\left\lceil\frac{\log _{2} u_{2}}{z u_{1}}\right\rceil-1$. $\left[u_{3} \leq 2^{z u_{1} u_{3}}-u_{3}-1\right.$ is always true by induction, since $z u_{1} \geq 4$ and $u_{2}=u_{3}$. The desired inequality now follows.]

(b) If $u_{2}<z<2 u_{2}$, then $\left(u_{1}, u_{2}, u_{3}, z\right)$ has a distinguishing 2-labeling as long as $z<2 u_{2} \leq 2^{u_{1} u_{2}^{2}}-\left\lceil\frac{\log _{2} u_{3}}{u_{1} u_{2}}\right\rceil-1$. [Since $u_{2}=u_{3}$, one can prove via induction that $2 u_{2} \leq 2^{u_{1} u_{2}^{2}}-u_{2}-1$ holds for $u_{2} \geq 3$. The desired inequality now follows.]

(ii) If $u_{4} \leq u_{2}^{2}$, then it is always true that $u_{4} \leq 2^{u_{2}^{2}}-\left\lceil\frac{\log _{2} u_{2}}{u_{2}}\right\rceil-1=2^{u_{2}^{2}}-2$. Hence, $\left(u_{2}, u_{2}, u_{4}\right)$ has a distinguishing 2-labeling. Therefore, Lemma 4.1 implies that $\left(u_{1}, u_{2}, u_{3}, u_{4}\right)$ has a distinguishing 2-labeling.

Case 4: $\left(u_{1}=u_{2}<u_{3} \leq u_{4}\right)$

(i) If $u_{2}^{2} \leq u_{3}$, then $u_{4} \leq c^{u_{3} u_{2}^{2}}-\left\lceil\frac{\log _{c} u_{3}}{u_{2}^{2}}\right\rceil-1$ implies that $\left(u_{2}^{2}, u_{3}, u_{4}\right)$ has a distinguishing $c$-labeling.

(ii) If $u_{3}<u_{2}^{2} \leq u_{4}$, then $u_{4} \leq c^{u_{3} u_{2}^{2}}-\left\lceil\frac{\log _{c} u_{2}^{2}}{u_{3}}\right\rceil-1$ implies that $\left(u_{3}, u_{2}^{2}, u_{4}\right)$ has a distinguishing $c$-labeling. If $c^{u_{3} u_{2}^{2}}-\left\lceil\frac{\log _{c} u_{2}^{2}}{u_{3}}\right\rceil-1<u_{4} \leq c^{u_{3} u_{2}^{2}}-\left\lceil\frac{\log _{c} u_{3}}{u_{2}^{2}}\right\rceil-1$, then switch in the last dimension and look at $\left\lceil\frac{\log _{c} u_{3}}{u_{2}^{2}}\right\rceil+1 \leq z<\left\lceil\frac{\log _{c} u_{2}^{2}}{u_{3}}\right\rceil+1$. Note that the last string of inequalities implies that $2 \leq z<3$. Thus the only value of $z$ that we need to consider is $z=2$.

Hence, we consider $\left(2, u_{1}, u_{2}, u_{3}\right)$. By induction on $u_{4},\left(2, u_{1}, u_{2}, u_{3}\right)$ has a distinguishing $c$-labeling as $u_{3}<u_{2}^{2} \leq c^{2 u_{2}^{2}}-\left\lceil\frac{\log _{c} u_{2}}{2 u_{2}}\right\rceil-1=c^{2 u_{2}^{2}}-2$.

(iii) If $u_{3} \leq u_{4}<u_{2}^{2}$, then $\left(u_{3}, u_{4}, u_{2}^{2}\right)$ has a distinguishing 2-labeling as $u_{2}^{2} \leq 2^{u_{3} u_{4}}-$ $\left\lceil\frac{\log _{2} u_{4}}{u_{3}}\right\rceil-1$. [The inequality we want follows from the inequality $u_{2}^{2}<2^{u_{2}^{2}}-u_{2}^{2}-1$.]

Case 5: $\left(u_{1} \leq u_{2}=u_{3}=u_{4}\right)$ and $\left(u_{1}=u_{2}=u_{3}<u_{4}\right)$

(i) For $\left(u_{1}<u_{2}=u_{3}=u_{4}\right)$, we note that $\left(u_{2}, u_{3}, u_{4}\right)$ has a distinguishing 2-labeling. Hence, by Lemma 4.1, $\left(u_{1}, u_{2}, u_{3}, u_{4}\right)$ has one too.

(ii) The case $\left(u_{1}=u_{2}=u_{3}=u_{4}\right)$ is already known.

(iii) For $\left(u_{1}=u_{2}=u_{3}<u_{3}+1\right)$, we note that $\left(u_{2}, u_{3}, u_{3}+1\right)$ has a distinguishing 2-labeling. Hence, by Lemma 4.1, $\left(u_{1}, u_{2}, u_{3}, u_{3}+1\right)$ has one too.

(iv) For $\left(u_{1}=u_{2}=u_{3}<u_{3}+\varepsilon\right), 2 \leq \varepsilon \leq u_{3}^{3}-u_{3}$, we collapse and consider $\left(u_{3}+\varepsilon, u_{3}^{3}\right)$. As we will show, $u_{3}^{3} \leq 2^{u_{3}+\varepsilon}-\left\lceil\log _{2}\left(u_{3}+\varepsilon\right)\right\rceil-1$ holds for all $u_{3} \geq 2$, except for $\left(u_{3}, \varepsilon\right)=(4,2)$ and $\left(u_{3}, \varepsilon\right)=(5,2)$. By Lemma 4.1, $(4,4,4,6)$ and $(5,5,5,7)$ both have distinguishing 2-labelings. For all other cases, we observe 
that $\left(u_{3}+\varepsilon, u_{3}^{3}\right)$ has a distinguishing 2-labeling by induction on dimension and hence $\left(u_{1}=u_{2}=u_{3}<u_{3}+\varepsilon\right)$ has a distinguishing 2-labeling by the collapsing lemma.

$\left[u_{3} \leq 2^{u_{3}+\varepsilon}-\left\lceil\log _{2}\left(u_{3}+\varepsilon\right)\right\rceil-1\right.$ can be verified by hand for the cases $2 \leq u_{3} \leq 9$. For $u_{3} \geq 10$, one can prove by induction that $u_{3}^{3} \leq 2^{u_{3}+1}-u_{3}^{3}-1$. Our result then follows.]

(v) For $\left(u_{1}=u_{2}=u_{3}<u_{3}^{3}+1\right)$ through $\left(u_{1}=u_{2}=u_{3}<c^{u_{3}^{3}}-\left\lceil\log _{c} u_{3}^{3}\right\rceil-1\right)$, we look at $\left(u_{3}^{3}, z\right), u_{3}^{3}+1 \leq z \leq c^{u_{3}^{3}}-\left\lceil\log _{c} u_{3}^{3}\right\rceil-1$.

(vi) The switching lemma takes care of $\left(u_{1}=u_{2}=u_{3}<c^{u_{3}^{3}}-\left\lceil\log _{c} u_{3}^{3}\right\rceil\right)$ through $\left(u_{1}=u_{2}=u_{3}<c^{u_{3}^{3}}-\left\lceil\frac{\log _{c} u_{3}}{u_{1} u_{2}}\right\rceil-1\right)$.

$d \geq 5$ : The case $(2,2,2,2, \ldots, 2)$ will serve as the basis step for induction on $u_{d}$.

Case 1: $\left(u_{1} \leq u_{2}<u_{3} \leq u_{4} \leq \cdots \leq u_{d-1} \leq u_{d}\right)$

(i) If $u_{1} u_{2} \leq u_{d-1}$, then $\left(u_{3}, u_{4}, \ldots, u_{1} u_{2}, \ldots, u_{d-1}, u_{d}\right)$ has a distinguishing $c$-labeling if $u_{d} \leq c^{u_{1} u_{2} \cdots u_{d-1}}-\left\lceil\frac{\log _{c} u_{d-1}}{u_{1} u_{2} \cdots u_{d-2}}\right\rceil-1$.

(ii) If $u_{d-1}<u_{1} u_{2} \leq u_{d}$, then $\left(u_{3}, u_{4}, \ldots, u_{d-1}, u_{1} u_{2}, u_{d}\right)$ has a distinguishing $c$ labeling if $u_{d} \leq c^{u_{1} u_{2} \cdots u_{d-1}}-\left\lceil\frac{\log _{c} u_{1} u_{2}}{u_{3} u_{4} \cdots u_{d-1}}\right\rceil-1$. Since $\left\lceil\frac{\log _{c} u_{1} u_{2}}{u_{3} u_{4} \cdots u_{d-1}}\right\rceil=\left\lceil\frac{\log _{c} u_{d-1}}{u_{1} u_{2} \cdots u_{d-2}}\right\rceil$ $=1$, we never have to worry about the case $c^{u_{1} u_{2} \cdots u_{d-1}}-\left\lceil\frac{\log _{c} u_{1} u_{2}}{u_{3} u_{4} \cdots u_{d-1}}\right\rceil-1<u_{d}$.

(iii) If $u_{d}<u_{1} u_{2}$, then $\left(u_{3}, u_{4}, \ldots, u_{d-1}, u_{d}, u_{1} u_{2}\right)$ has a distinguishing $c$-labeling as $u_{1} u_{2} \leq c^{u_{3} u_{4} \cdots u_{d}}-\left\lceil\frac{\log _{c} u_{d}}{u_{3} u_{4} \cdots u_{d-1}}\right\rceil-1=c^{u_{3} u_{4} \cdots u_{d}}-2$. Because $u_{d}<u_{1} u_{2}$, we do not have to worry about the case $c^{u_{3} u_{4} \cdots u_{d}}-\left\lceil\frac{\log _{c} u_{d}}{u_{3} u_{4} \cdots u_{d-1}}\right\rceil-1 \leq u_{d}$.

Case 2: $\left(u_{1}=u_{2}=\cdots=u_{k}<u_{k+1} \leq \cdots \leq u_{d-1} \leq u_{d}\right)$, where $2 \leq k \leq d-2$

(i) If $u_{1}^{k} \leq u_{d-1}$, then $\left(u_{k+1}, \ldots, u_{1}^{k}, \ldots, u_{d-1}, u_{d}\right)$ has a distinguishing $c$-labeling if $u_{d} \leq c^{u_{1}^{k} \cdots u_{d-1}}-\left\lceil\frac{\log _{c} u_{d-1}}{u_{1}^{k} \cdots u_{d-2}}\right\rceil-1$.

(ii) If $u_{d-1}<u_{1}^{k} \leq u_{d}$, then $\left(u_{k+1}, \ldots, u_{d-1}, u_{1}^{k}, u_{d}\right)$ has a distinguishing $c$-labeling if $u_{d} \leq c^{u_{1}^{k} \cdots u_{d-1}}-\left\lceil\frac{\log _{c} u_{1}^{k}}{u_{k+1} \cdots u_{d-1}}\right\rceil-1$. If $c^{u_{1}^{k} \cdots u_{d-1}}-\left\lceil\frac{\log _{c} u_{1}^{k}}{u_{k+1} \cdots u_{d-1}}\right\rceil-1<u_{d} \leq$ $c^{u_{1}^{k} \cdots u_{d-1}}-\left\lceil\frac{\log _{c} u_{d-1}}{u_{1} u_{2} \cdots u_{d-2}}\right\rceil-1=c^{u_{1}^{k} \cdots u_{d-1}}-2$, then switch and look at

$$
2=\left\lceil\frac{\log _{c} u_{d-1}}{u_{1} u_{2} \cdots u_{d-2}}\right\rceil+1 \leq z<\left\lceil\frac{\log _{c} u_{1}^{k}}{u_{k+1} \cdots u_{d-1}}\right\rceil+1=k+1<u_{1}^{k} .
$$

(a) If $z<u_{d-1}$, then look at $\left(u_{1}, u_{2}, \ldots, z, \ldots, u_{d-1}\right)$. Note that $\left(u_{1}, u_{2}, \ldots, z\right.$, $\left.\ldots, u_{d-1}\right)$ has a distinguishing $c$-labeling if $u_{d-1} \leq c^{u_{1}^{k} u_{k+1} \cdots z}-\left\lceil\frac{\log _{c} \delta}{\varepsilon}\right\rceil-1$, where $\varepsilon=u_{1} u_{2} \cdots u_{d-2}$ if $\delta=z$ and $\varepsilon=u_{1} u_{2} \cdots u_{d-3} z$ if $\delta=u_{d-2}$. Since, in either case, $\left\lceil\frac{\log _{c} \delta}{\varepsilon}\right\rceil \leq u_{d-1}$, it follows that $u_{d-1} \leq c^{u_{1}^{k} u_{k+1} \cdots z}-\left\lceil\frac{\log _{c} \delta}{\varepsilon}\right\rceil-1$ always holds.

(b) If $u_{d-1} \leq z<u_{1}^{k}$, then we consider $\left(u_{1}, u_{2}, \ldots, u_{k}, u_{k+1}, \ldots, u_{d-1}, z\right)$. Since it is always true that $z \leq c^{u_{1}^{k} u_{k+1} \cdots u_{d-1}}-\left\lceil\frac{\log _{c} u_{d-1}}{u_{1}^{k} \cdots u_{d-2}}\right\rceil-1=c^{u_{1}^{k} u_{k+1} \cdots u_{d-1}}-2$, $\left(u_{1}, u_{2}, \ldots, u_{k}, u_{k+1}, \ldots, u_{d-1}, z\right)$ has a distinguishing $c$-labeling. 
(iii) If $u_{d}<u_{1}^{k}$, then $u_{d} \leq 2^{u_{1}^{k} u_{k+1} \cdots u_{d-2}}-2$. Thus, $\left(u_{1}, u_{2}, \ldots, u_{d-2}, u_{d}\right)$ has a distinguishing 2-labeling and so, by Lemma $4.1,\left(u_{1}, u_{2}, \ldots, u_{d-2}, u_{d-1}, u_{d}\right)$ has one too.

Case 3: $\left(u_{1}<u_{2}=\cdots=u_{k}<u_{k+1} \leq \cdots \leq u_{d-1} \leq u_{d}\right)$, where $3 \leq k \leq d-1$

(i) If $u_{2}^{k-1} \leq u_{d-1}$, then $\left(u_{1}, u_{k+1}, \ldots, u_{2}^{k-1}, \ldots, u_{d-1}, u_{d}\right)$ has a distinguishing $c$ labeling if $u_{d} \leq c^{u_{1} u_{2}^{k-1} \cdots u_{d-1}}-\left\lceil\frac{\log _{c} u_{d-1}}{u_{1} u_{2}^{k-1} \cdots u_{d-2}}\right\rceil-1$.

(ii) If $u_{d-1}<u_{2}^{k-1} \leq u_{d}$, then $\left(u_{1}, u_{k+1}, \ldots, u_{d-1}, u_{2}^{k-1}, u_{d}\right)$ has a distinguishing $c$-labeling if $u_{d} \leq c^{u_{1} u_{2}^{k-1} \cdots u_{d-1}}-\left\lceil\frac{\log _{c} u_{2}^{k-1}}{u_{k+1} \cdots u_{d-1}}\right\rceil-1$.

If $c^{u_{1} u_{2}^{k-1} \cdots u_{d-1}}-\left\lceil\frac{\log _{c} u_{2}^{k-1}}{u_{k+1} \cdots u_{d-1}}\right\rceil-1<u_{d} \leq c^{u_{1} u_{2}^{k-1} \cdots u_{d-1}}-\left\lceil\frac{\log _{c} u_{d-1}}{u_{1} u_{2} \cdots u_{d-2}}\right\rceil-1$, then switch and look at $2=\left\lceil\frac{\log _{c} u_{d-1}}{u_{1} u_{2} \cdots u_{d-2}}\right\rceil+1 \leq z<\left\lceil\frac{\log _{c} u_{2}^{k-1}}{u_{k+1} \cdots u_{d-1}}\right\rceil+1 \leq k<u_{2}^{k-1}$.

(a) If $z<u_{d-1}$, then consider $\left(u_{1}, u_{2}, \ldots, u_{k}, u_{k+1}, \ldots, z, \ldots, u_{d-1}\right)$. Note that $\left(u_{1}, u_{2}, \ldots, u_{k}, u_{k+1}, \ldots, z, \ldots, u_{d-1}\right)$ has a distinguishing $c$-labeling if $u_{d-1} \leq$ $c^{u_{1} u_{2}^{k-1} \cdots u_{d-2} z}-\left\lceil\frac{\log _{c} \delta}{\varepsilon}\right\rceil-1$, where $\varepsilon=u_{1} u_{2} \cdots u_{d-2}$ if $\delta=z$ and $\varepsilon=$ $u_{1} u_{2} \cdots u_{d-3} z$ if $\delta=u_{d-2}$. Since, in either case, $\left\lceil\frac{\log _{c} \delta}{\varepsilon}\right\rceil \leq u_{d-1}$, it follows that $u_{d-1} \leq c^{u_{1} u_{2}^{k-1} \cdots u_{d-2} z}-\left\lceil\frac{\log _{c} \delta}{\varepsilon}\right\rceil-1$ always holds.

(b) If $u_{d-1} \leq z<u_{2}^{k-1}$, then look at $\left(u_{1}, u_{2}, \ldots, u_{k}, u_{k+1}, \ldots, u_{d-1}, z\right)$. Since $z \leq c^{u_{1} u_{2}^{k-1} \cdots u_{d-1}}-\left\lceil\frac{\log _{c} u_{d-1}}{u_{1} u_{2}^{k-1} \cdots u_{d-2}}\right\rceil-1=c^{u_{1} u_{2}^{k-1} \cdots u_{d-1}}-2$ always holds, $\left(u_{1}, u_{2}, \ldots, u_{k}, u_{k+1}, \ldots, u_{d-1}, z\right)$ has a distinguishing $c$-labeling by induction on $u_{d}$.

(iii) If $u_{d}<u_{2}^{k-1}$, then $u_{d} \leq 2^{u_{2}^{k-1}} u_{k+1} \cdots u_{d-1}-2$. Thus $\left(u_{2}, u_{3}, \ldots, u_{d}\right)$ has a distinguishing 2-labeling and so, by Lemma $4.1,\left(u_{1}, u_{2}, \ldots, u_{d}\right)$ has one too.

Case 4: $\left(u_{1} \leq u_{2}=\cdots=u_{d-1}=u_{d}\right)$ and $\left(u_{1}=u_{2}=\cdots=u_{d-1}<u_{d}\right)$

(i) For $\left(u_{1} \leq u_{2}=u_{3}=\cdots=u_{d-1}=u_{d}\right)$, we note that $\left(u_{2}, u_{3}, \ldots, u_{d}\right)$ has a distinguishing 2-labeling and so, by Lemma $4.1,\left(u_{1}, u_{2}, \ldots, u_{d}\right)$ has one too.

(ii) For $\left(u_{1}=u_{2}=\cdots=u_{d-1}<u_{d}\right), u_{1}<u_{d}<u_{1}^{d-2}$, we note that $u_{d}<u_{1}^{d-1}$. Therefore, since $\left(u_{1}, u_{2}, \ldots, u_{d-1}\right)$ has a distinguishing 2-labeling, Lemma $4.1 \mathrm{im}$ plies that $\left(u_{1}, u_{2}, \ldots, u_{d-1}, u_{d}\right)$ also has a distinguishing 2-labeling.

(iii) If $u_{1}^{d-2} \leq u_{d}<u_{1}^{d-1}$, then $\left(u_{d}, u_{1}^{d-1}\right)$ has a distinguishing 2-labeling since $u_{1}^{d-1} \leq$ $2^{u_{1}^{d-2}}-u_{1}^{d-1}-1 \leq 2^{u_{d}}-u_{d}-1$.

(iv) If $u_{1}^{d-1} \leq u_{d} \leq c^{u_{1}^{d-1}}-\left\lceil\log _{c} u_{1}^{d-1}\right\rceil-1$, then $\left(u_{1}^{d-1}, u_{d}\right)$ has a distinguishing $c$-labeling (since $\left.u_{d} \leq c^{u_{1}^{d-1}}-\left\lceil\log _{c} u_{1}^{d-1}\right\rceil-1\right)$.

(v) Lastly, if $c^{u_{1}^{d-1}}-\left\lceil\log _{c} u_{1}^{d-1}\right\rceil \leq u_{d} \leq c^{u_{1}^{d-1}}-\left\lceil\frac{\log _{c} u_{d-1}}{u_{1}^{d-2}}\right\rceil-1=c^{u_{1}^{d-1}}-2$, then we switch and look at $\left(z, u_{1}, u_{2}, \ldots, u_{d-1}\right)$ or $\left(u_{1}, u_{2}, \ldots, u_{d-1}, z\right)$, where $2 \leq z \leq$ $\left\lceil\log _{c} u_{1}^{d-1}\right\rceil<u_{1}^{d-1}$. In either case, it follows that $\left(u_{1}, u_{2}, \ldots, u_{d-1}, u_{d}\right)$ has a distinguishing $c$-labeling. 
Lemma 4.3. For integers $c \geq 2, d \geq 3$ and $1 \leq u_{1} \leq u_{2} \leq \cdots \leq u_{d}$ with $u_{d} \geq$ $c^{u_{1} u_{2} \cdots u_{d-1}}-\left\lceil\frac{\log _{c} u_{d-1}}{u_{1} u_{2} \cdots u_{d-2}}\right\rceil+1$, there is no distinguishing $c$-labeling of the $\left(u_{1}, u_{2}, \ldots, u_{d}\right)$ grid.

Proof. If $u_{d}>c^{u_{1} u_{2} \cdots u_{d-1}}$, then since the dimension $d$ subgrids have size $\left(u_{1}, u_{2}, \ldots\right.$, $\left.u_{d-1}\right)$ and there are greater than $c^{u_{1} u_{2} \cdots u_{d-1}}$ such subgrids, at least two of them must be identical. An automorphism switching two such positions in dimension $d$ preserves labels. So, there is no distinguishing $c$-labeling in this case.

If $u_{d}=c^{u_{1} u_{2} \cdots u_{d-1}}$ and two dimension $d$ subgrids are identical, apply the same automorphism as the previous paragraph. Otherwise, the set of dimension $d$ subgrids is exactly the set of possible subgrids. Then, take any nontrivial automorphisms $\pi_{1}, \ldots, \pi_{d-1}$ in the other dimensions. This induces a permutation $\pi_{d}$ of the dimension $d$ subgrids. Then, $\left(\pi_{1}, \ldots, \pi_{d}\right)$ preserves labels. So, there is no distinguishing $c$-labeling in this case.

If $c^{u_{1} u_{2} \cdots u_{d-1}}-\left\lceil\frac{\log _{c} u_{d-1}}{u_{1} u_{2} \cdots u_{d-2}}\right\rceil+1 \leq u_{d}<c^{u_{1} u_{2} \cdots u_{d-1}}$, by the grid switching lemma there is a distinguishing $c$-labeling only if there is a distinguishing $c$-labeling of the grid of size $\left(u_{1}, u_{2}, \ldots, u_{d-1}, c^{u_{d}}-z\right)$, where $1 \leq z \leq\left\lceil\frac{\log _{c} u_{d-1}}{u_{1} u_{2} \cdots u_{d-2}}\right\rceil-1$. Note that $u_{d-1} \geq z$ and that $z \cdot\left(u_{1} \cdots u_{d-2}\right)<\left(\frac{\log _{c} u_{d-1}}{u_{1} u_{2} \cdots u_{d-2}}\right) \cdot\left(u_{1} \cdots u_{d-2}\right)=\log _{c} u_{d-1}$. So, $c^{z \cdot\left(u_{1} \cdots u_{d-2}\right)}<$ $c^{\log _{c} u_{d-1}}=u_{d-1}$. Hence, by the first paragraph, there is no distinguishing $c$-labeling.

Theorem 4.4. For integers $d \geq 2$ and $1 \leq u_{1} \leq \cdots \leq u_{d}$ let $s=u_{1} u_{2} \cdots u_{d-1}$ and $c=\left\lceil u_{d}^{1 / s}\right\rceil$. Except for $d=2$ with $u_{1}=u_{2} \in\{2,3\}$ and for $d=3$ with $u_{1}=u_{2}=$ $u_{3}=2$ we have that the grid and array of size $\left(u_{1}, u_{2}, \ldots, u_{d}\right)$ have a distinguishing $c$ labeling if $u_{d} \leq c^{u_{1} u_{2} \cdots u_{d-1}}-\left\lceil\frac{\log _{c} u_{d-1}}{u_{1} u_{2} \cdots u_{d-2}}\right\rceil-1$. They have no distinguishing $c$-labeling if $u_{d} \geq c^{u_{1} u_{2} \cdots u_{d-1}}-\left\lceil\frac{\log _{c} u_{d-1}}{u_{1} u_{2} \cdots u_{d-2}}\right\rceil+1$.

Proof. If the array has a distinguishing c-labeling, so does the grid. If the grid has no distinguishing $c$-labeling, neither does the array. The result then follows immediately from Lemmas 4.2 and 4.3 .

In the remaining case, $u_{d} \geq c^{u_{1} u_{2} \cdots u_{d-1}}-\left\lceil\frac{\log _{c} u_{d-1}}{u_{1} u_{2} \cdots u_{d-2}}\right\rceil$, the theorem gives no information. However, in these cases, we can apply the switching lemma to check. We again may need to recursively check the new sizes. However, the number of such steps will be at most the iterated logarithm (base $c$ ) of $u_{d}$.

\section{Distinguishing numbers}

In this section, we take the results of the previous section in order to determine (up to two possible values) the distinguishing numbers for arrays and grids.

Theorem 4.4 was stated from the perspective of whether or not a distinguishing $c$ labeling exists for given $c$ and $\left(u_{1}, u_{2}, \ldots, u_{d}\right)$. For given $\left(u_{1}, u_{2}, \ldots, u_{d}\right)$, the distinguishing number (for a grid or array) is the smallest $c$ such that the condition for a distinguishing labeling in Theorem 4.4 is satisfied. Determining this $c$ we get the following result for distinguishing numbers.

Corollary 5.1. For integers $d \geq 2$ and $1 \leq u_{1} \leq \cdots \leq u_{d}$, let $s=u_{1} u_{2} \cdots u_{d-1}$ and $c=\left\lceil u_{d}^{1 / s}\right\rceil$. Except for $d=2$ with $u_{1}=u_{2} \in\{2,3\}$ and for $d=3$ with $u_{1}=u_{2}=$ $u_{3}=2$, we have $D\left(\operatorname{Grid}\left(u_{1}, u_{2}, \ldots, u_{d}\right)\right)=D\left(\operatorname{Array}\left(u_{1}, u_{2}, \ldots, u_{d}\right)\right) \in\{c, c+1\}$. 
It is $c$ if $u_{d} \leq c^{s}-\left\lceil\frac{\log _{c} u_{d-1}}{u_{1} u_{2} \cdots u_{d-2}}\right\rceil-1$ and $c+1$ if $c^{s}-\left\lceil\frac{\log _{c} u_{d-1}}{u_{1} u_{2} \cdots u_{d-2}}\right\rceil+1 \leq u_{d}$. When $u_{d}=c^{s}-\left\lceil\frac{\log _{c} u_{d-1}}{u_{1} u_{2} \cdots u_{d-2}}\right\rceil$, it is $c$ if the grid of size $\left(u_{1}, u_{2}, \ldots, c^{s}-u_{d}\right)$ has a distinguishing $c$-labeling and $c+1$ otherwise. Hence, we can recursively determine the value in this case.

Proof. It is easy to check that for $k<c, u_{d} \geq k^{s}-\left\lceil\frac{\log _{k} u_{d-1}}{u_{1} u_{2} \cdots u_{d-2}}\right\rceil+1$ and that $u_{d} \leq$ $(c+1)^{s}-\left\lceil\frac{\log _{c+1} u_{d-1}}{u_{1} u_{2} \cdots u_{d-2}}\right\rceil-1$. Hence, the smallest $c$ such that there is a distinguishing $c$-labeling is $c$ or $c+1$. The exact values follow immediately from Theorem 4.4. The comment about the recursion follows from the switching lemma.

We finish with a few comments and examples. The distinguishing numbers for the exceptional cases in the corollary are 2 for grids and 3 for arrays as noted earlier. For the special cases when we use recursion, it is possible that the smaller grid/array has a distinguishing number smaller than $c$. However, what matters is whether or not the reduced case has a distinguishing $c$-labeling. Consider the following sizes for examples with $d=3$ : $\left(3,5^{6}-2,5^{3 \cdot\left(5^{6}-2\right)}-2\right),\left(3,5^{6}-1,5^{3 \cdot\left(5^{6}-1\right)}-2\right),\left(3,5^{6}, 5^{3 \cdot 5^{6}}-2\right)$. In each case, $c=5$ and we are on the boundary where we need to apply recursion. By the switching lemma, we look at (respectively) 5-labelings for sizes $\left(2,3,5^{6}-2\right),\left(2,3,5^{6}-1\right),\left(2,3,5^{6}\right)$. In the first case, the conditions give a 5 -labeling and in the last case they do not. So $D\left(\operatorname{Grid}\left(3,5^{6}-\right.\right.$ $\left.2,5^{3 \cdot\left(5^{6}-2\right)}-2\right)=5$ and $D\left(\operatorname{Grid}\left(3,5^{6}, 5^{3 \cdot 5^{6}}-2\right)=6\right.$. In the middle case we again are on a boundary and apply the switching lemma one more time, looking at size $(1,2,3)$. Here, there is a distinguishing 5-labeling so we get $D\left(\operatorname{Grid}\left(3,5^{6}-1,5^{3 \cdot\left(5^{6}-1\right)}-2\right)=5\right.$. It happens also that $D\left(\operatorname{Grid}\left(2,3,5^{6}-1\right)=5\right.$, but it could have been lower. Note that $D(\operatorname{Grid}(1,2,3)=2$, but the fact that there is a distinguishing 2-labeling does not help for size $\left(2,3,5^{6}-1\right)$ as we need at least 5 labels for this size.

Acknowledgements We would like to thank the anonymous referees who made useful comments that improved the paper. In particular, one referee suggested the comments in the last three paragraphs of section 1. In addition, we would also like to posthumously acknowledge Mike Albertson who gave very useful advice during the preparation of our paper [5] giving a 2-dimensional result, as well as preliminary discussions on the results in this paper.

\section{References}

[1] M. O. Albertson, Distinguishing Cartesian powers of graphs, Electron. J. Combin. 12 (2005), Note \#17.

[2] M. O. Albertson and K. L. Collins, Symmetry breaking in graphs, Electron. J. Combin. 3 (1996), Research Paper \#18.

[3] B. Bogstad and L. J. Cowen, The distinguishing number of the hypercube, Discrete Math. 283 (2004), 29-35.

[4] M. Chan, The distinguishing number of the direct product and wreath product action, J. Algebraic Combin. 24 (2006), 331-345.

[5] M. J. Fisher and G. Isaak, Distinguishing colorings of Cartesian products of complete graphs, Discrete Math. 308 (2008), 2240-2246.

[6] F. Harary and D. Ranjan, Identity orientations of complete bipartite graphs, Discrete Math. 290 (2005), 173-182. 
[7] W. Imrich, J. Jerebic and S. Klavžar, The distinguishing number of Cartesian products of complete graphs, European J. Combin. 29 (2008), 922-929.

[8] W. Imrich and S. Klavžar, Product Graphs, John Wiley \& Sons, Inc., New York, 2000.

[9] W. Imrich and S. Klavžar, Distinguishing Cartesian powers of graphs, Distinguishing Cartesian powers of graphs, J. Graph Theory 53 (2006), 250-260.

[10] S. Klavžar and X. Zhu, Cartesian powers of graphs can be distinguished with two labels, European J. Combin. 28 (2007), 303-310. 